\title{
Genomic sequence and activity of KSI 0 , a transposable phage of the Burkholderia cepacia complex
}

\author{
Amanda D Goudie ${ }^{1}$, Karlene H Lynch ${ }^{1}$, Kimberley D Seed ${ }^{1}$, Paul Stothard ${ }^{2}$, \\ Savita Shrivastava ${ }^{3}$, David S Wishart ${ }^{1,3}$ and Jonathan J Dennis*1
}

Address: ${ }^{1}$ Department of Biological Sciences, University of Alberta, Edmonton, Alberta, Canada, ${ }^{2}$ Department of Agricultural, Food and Nutritional Science, University of Alberta, Edmonton, Alberta, Canada and ${ }^{3}$ Department of Computing Science, University of Alberta, Edmonton, Alberta, Canada

Email: Amanda D Goudie - agoudie@ualberta.ca; Karlene H Lynch - khlynch@ualberta.ca; Kimberley D Seed - kseed@ualberta.ca; Paul Stothard - paul.stothard@ualberta.ca; Savita Shrivastava - savita@ualberta.ca; David S Wishart - david.wishart@ualberta.ca; Jonathan J Dennis* - jon.dennis@ualberta.ca

* Corresponding author

Published: 18 December 2008

BMC Genomics 2008, 9:615 doi:10.1/86/147|-2164-9-615
Received: 26 June 2008

Accepted: 18 December 2008

This article is available from: http://www.biomedcentral.com//47/-2/64/9/6/5

(c) 2008 Goudie et al; licensee BioMed Central Ltd.

This is an Open Access article distributed under the terms of the Creative Commons Attribution License (http://creativecommons.org/licenses/by/2.0), which permits unrestricted use, distribution, and reproduction in any medium, provided the original work is properly cited.

\begin{abstract}
Background: The Burkholderia cepacia complex $(B C C)$ is a versatile group of Gram negative organisms that can be found throughout the environment in sources such as soil, water, and plants. While BCC bacteria can be involved in beneficial interactions with plants, they are also considered opportunistic pathogens, specifically in patients with cystic fibrosis and chronic granulomatous disease. These organisms also exhibit resistance to many antibiotics, making conventional treatment often unsuccessful. $\mathrm{KSIO}$ was isolated as a prophage of $B$. cenocepacia $\mathrm{K} 56-2$, a clinically relevant strain of the BCC. Our objective was to sequence the genome of this phage and also determine if this prophage encoded any virulence determinants.
\end{abstract}

Results: KSIO is a 37,635 base pairs (bp) transposable phage of the opportunistic pathogen Burkholderia cenocepacia. Genome sequence analysis and annotation of this phage reveals that KSIO shows the closest sequence homology to Mu and BcepMu. KSIO was found to be a prophage in three different strains of B. cenocepacia, including strains K56-2, J2315, and C5424, and seven tested clinical isolates of $B$. cenocepacia, but no other BCC species. A survey of 23 strains and 20 clinical isolates of the BCC revealed that KSIO is able to form plaques on lawns of B. ambifaria LMG 19467, B. cenocepacia PCI84, and B. stabilis LMG 18870.

Conclusion: $\mathrm{KSIO}$ is a novel phage with a genomic organization that differs from most phages in that its capsid genes are not aligned into one module but rather separated by approximately II kb, giving evidence of one or more prior genetic rearrangements. There were no potential virulence factors identified in KSIO, though many hypothetical proteins were identified with no known function.

\section{Background}

The Burkholderia cepacia complex (BCC) is a group of Gram negative, motile bacilli, first described in 1950 by W.H. Burkholder as the causative agent of soft rot in onion [1]. BCC species can be found throughout the environment, and have been isolated from sources such as soil, water, and plants [2]. BCC organisms are extremely diverse and versatile in their metabolic capabilities and 
although discovered as a plant pathogen, some strains of the BCC are actually beneficial to plants and to the environment. The BCC has also become known as an important group of opportunistic pathogens in immunocompromised patients, specifically in those with cystic fibrosis (CF) or chronic granulomatous disease (CGD) $[3,4]$. Since most clinically relevant strains of the $\mathrm{BCC}$ are resistant to multiple antibiotics, the most effective treatments against BCC bacteria involve specific antibiotic combinations. However, even with repeated combination antibiotic therapy in CF patients, clearance of the microorganisms is not observed. As an alternative treatment, bacteriophage (or phage) therapy is currently being researched and tested for the treatment of BCC infections [5].

Phage therapy, developed Felix d'Herelle, involves the use of lytic phages to kill infecting bacteria [6]. However, modified temperate phages or phage products may also be considered [7]. Phage therapy may prove to be more effective and efficient than antibiotics, especially since it is much easier to modify a phage than it is to develop a new antibiotic when bacterial strains become resistant. However, there are still a number of problems with using phages as therapeutic agents that must be overcome, one of these problems being lysogenic conversion. In order for phage therapy to be used safely, especially when involving temperate phages, it is important to determine if phages encode bacterial toxins or genes that could be harmful if acquired by the host bacterium. A Mu-like phage of the BCC, BcepMu, for example, encodes a putative ExeA homolog, which may be involved in the secretion of toxins, and a 3-O-acyltransferase homolog, which may be responsible for a bacterium's resistance towards certain antibiotics [8]. Other well-known examples of phages conferring virulence to their bacterial hosts are the cholera toxin of Vibrio cholera, the Shiga toxin of E. coli, and the scarlet fever toxin of Streptococcus pyogenes [9-11]. Therefore, phages should be sequenced to ensure that they do not harbor potential virulence determinants before they are used in a therapeutic setting.

In 2005, Seed and Dennis isolated four lytic phages from onion rhizosphere as well as five temperate phages from five different strains within the BCC [5]. One of these temperate phages, KS10, was isolated as prophage of $B$. cenocepacia strain K56-2. Most B. cenocepacia of ET12 lineage similar to K56-2, such as strain J2315, harbour a Mu-like prophage called BcepMu, while K56-2 does not [8]. A search of the previously determined $B$. cenocepacia J2315 genomic sequence, as well as a more extensive search for lysogeny in BCC strains, determined BcepMu to be the only known prophage of $\mathrm{J} 2315$ [12]. This study reports the host range, genome sequence and organization, and putative gene functions of the transposable phage KS10 in comparison to BcepMu and $\mathrm{Mu}$. Analysis of the KS10 genome will allow us to identify any potential virulence determinants encoded by this temperate BCC phage.

\section{Results and Discussion \\ Properties of lysogenic phage KSIO}

KS10 was originally identified as pinpoint plaques present on lawns of uninduced cultures of B. cenocepacia K56-2. In contrast to phage $\mathrm{Mu}$, and many other Mu-like phages, KS10 in K56-2 appears to spontaneously switch to its lytic life cycle at a high frequency. Because of this, it was not necessary to use mitomycin $\mathrm{C}$ or exposure to UV light to initiate induction. The phage lysate from $B$. cenocepacia K56-2 plated on another BCC strain well-known to be susceptible to phage infection [5], B. ambifaria LMG 19467, produces slightly larger plaques than the small pinpoint plaques found on K56-2. To confirm that KS10 is a prophage of K56-2, KS10 was propagated on LMG 19467 and did not form plaques on K56-2, indicating resistance to superinfection. KS10 particles are structurally stable over a long period of time, with titers remaining as high as $6.75 \times 10^{6} \mathrm{pfu} / \mathrm{ml}$ after storage in suspension media (SM) for 10 months. Because of this trait, just one plaque of KS10 in $1 \mathrm{~mL}$ of SM can produce confluent lysis when plated with strain LMG 19467.

An electron micrograph (EM) of KS10 virions negatively stained with $2 \%$ phosphotungstic acid shows an icosahedral head and tail that appears to be contractile upon visualization (Figure 1). This is typical of the Myoviridae family of phages. EM analysis also reveals the average KS10 head size to be approximately $80 \mathrm{~nm}$ with a tail length of approximately $140 \mathrm{~nm}$.

\section{Determination of the genome sequence}

The KS10 DNA sequence was determined using a shotgun cloning and sequencing approach. Phage DNA was digested with restriction enzymes, ligated into pUC19 or pGEM7Z, and transformed into chemically competent DH5 $\alpha$ cells. Inserts larger than $10 \mathrm{~kb}$ were also subcloned and sequenced. All inserts were sequenced at least twice, and PCR or primer walking was used to fill gaps in the sequence. Initial restriction digests suggested an estimated genome size of $35 \mathrm{~kb}$. Approximately 249 runs with an average read-length of 680 bp were assembled to give greater then 4-fold genome coverage, resulting in a single contig totaling 37,635 bp in length. This DNA sequence had approximately $63 \%$ GC content, which is significantly higher than that of phage $\mathrm{Mu}$, but similar to BcepMu. In order to determine if the recently completed B. cenocepacia J2315 genome was lysogenized by KS10, a BLASTX analysis was carried out at the Wellcome Trust Sanger Institute site http://www.sanger.ac.uk/cgi-bin/ blast/submitblast/b_cenocepacia, revealing that KS10 is indeed a prophage of B. cenocepacia J2315. A comparison 

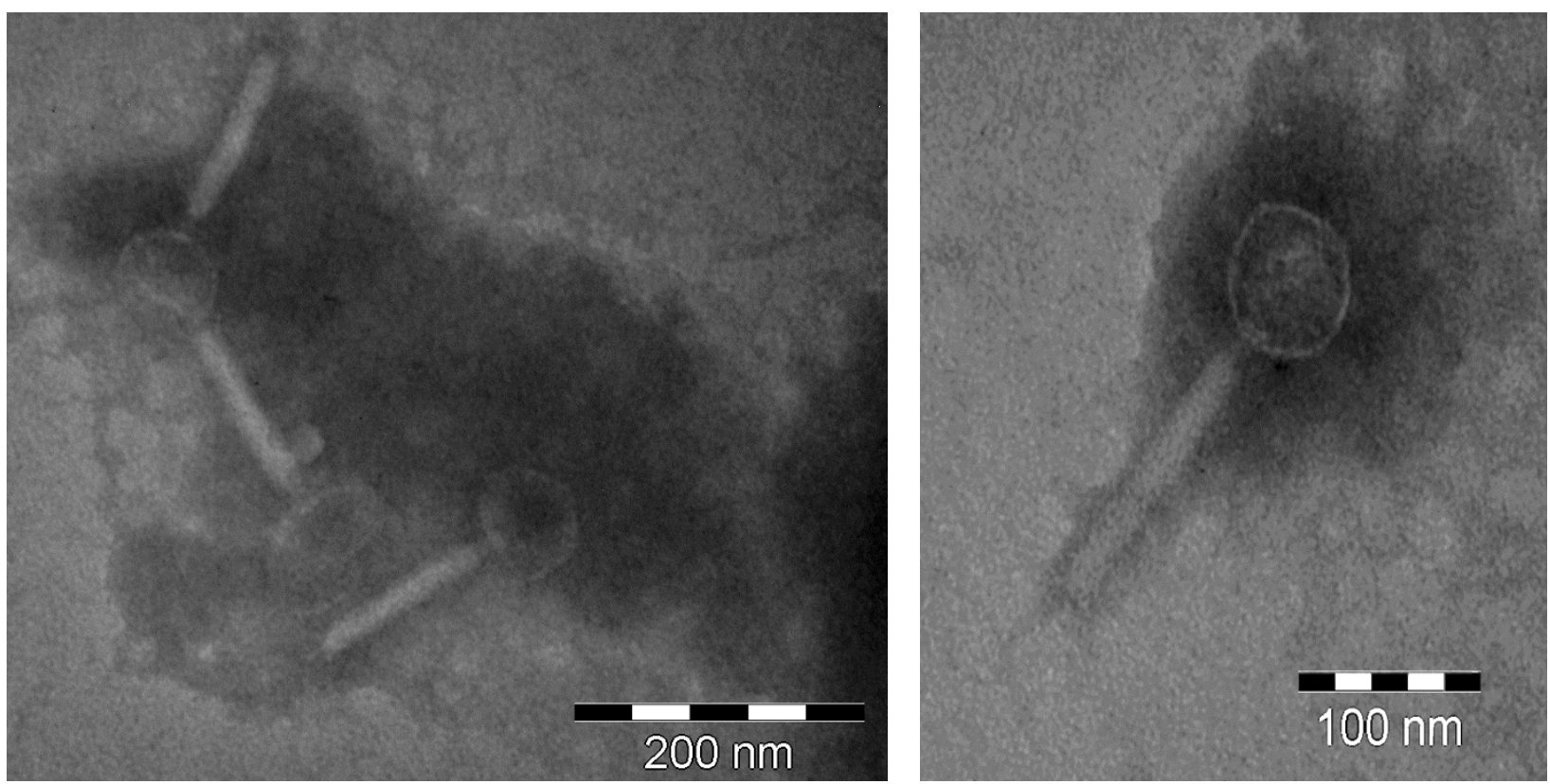

\section{Figure I}

Electron micrographs showing morphology of KSIO particles in lysate stored at $4^{\circ} \mathrm{C}$ for approximately one week prior to imaging. Lysate was negatively stained with $2 \%$ phosphotungstic acid. Images were viewed at II 0,000 -fold magnification (A), and I40,000-fold magnification (B) using a Philips/FEl (Morgagni) Transmission Electron Microscope with CCD camera in the Biological Science Department's Microscopy Unit.

of the KS10 sequence with the J2315 genomic sequence showed an exact KS10 sequence is located in chromosome 1 of J2315 (bp 1,766,551 to bp 1,728,918), on the complementary strand. As B. cenocepacia J2315 also harbours prophage BcepMu, this strain contains two functionally similar Mu-like phages, whereas B. cenocepacia K56-2 only contains the Mu-like phage KS10.

The right end phage insertion site, which varies across and within $B$. cenocepacia strains, was determined by sequencing two clones and one subclone from B. cenocepacia K56-2 that contained the phage/host DNA junction at two different insertion sites. These results indicated that there was random insertion occurring within the B. cenocepacia K56-2 genome, a feature characteristic of transposable phages. The left end phage insertion site was obtained using PCR of genomic DNA since no shotgun clones containing a phage/host insert were originally isolated. Forward primers were designed to the chromosome for the region upstream of the gene interrupted by the KS10 phage as determined for the right end insertion site, and reverse primers were designed to the left end of completed KS10 phage sequence.

\section{Host range and presence of $K S I O$ within the $B C C$}

Using a plaque assay, 23 BCC strains were tested for their sensitivity to KS10. Only three of the tested strains were found to support plaque formation with KS10: B. ambifaria LMG 19467, B. cenocepacia PC184, and B. stabilis LMG 18870. Lysates obtained from filter sterilized uninduced overnight cultures of both K56-2 and J2315 were able to form plaques on LMG 19467, PC184, and LMG 18870. Since B. ambifaria LMG 19467 (but not B. cenocepacia PC184) is also a host for BcepMu, lysate obtained from an overnight culture of $B$. cenocepacia J2315 will also form BcepMu plaques on a lawn of $B$. ambifaria LMG 19467. On these plates we observed a noticeable increase in the number of plaques, suggesting that both KS10 and BcepMu were being shed by J2315. It is possible that KS10 was not detected in previous attempts to isolate prophages from strain J2315 by assaying an overnight culture for PFU [12] due to its relatively poor host range, and its overlap in host range with BcepMu. Interestingly, although the lysogenic phase phage repressors of KS10 and BcepMu show 36\% identity to each other, and both prophages are found integrated into B. cenocepacia strains such as J2315, in B. cenocepacia K56-2 only KS10 can exist as a prophage while BcepMu will form plaques on this strain.

In host K56-2, at the time of initial KS10 sequencing, the majority of the cloned host/phage sequences show that KS10 was inserted before amino acid 394 of a transcrip- 
tional regulator in the GntR family (Table 1). Since KS10 is a transposable phage, after transposition it can be inserted in many different locations within the genome. BLASTN analysis of the host DNA flanking the phage sequence from the Sanger site indicates that in B. cenocepacia $\mathrm{J} 2315$ the prophage is located in chromosome 1, and has inserted in an oxidoreductase gene in the Gfo/Idh/ MocA family around amino acid 235. It has been suggested that prophages are arranged so that their structural genes are oriented in the same direction as the genes surrounding them, but the orientation of the KS10 prophage in J2315 orients the transcription of structural genes from right to left, while the surrounding genes are transcribed from left to right (Figure 2) $[13,14]$.

To determine the distribution of KS10 within the genome of other BCC species/strains, PCR using primers specific to the KS10 sequence was used. B. cenocepacia K56-2 and J2315 chromosomes were used as positive controls since these strains are known to be lysogenized by KS10. B. ambifaria LMG 19467 genomic DNA was used as a negative control due to the ability of KS10 to form plaques on this strain. Seven strains of B. cenocepacia and one strain from each of the other species in the BCC experimental strain panel were tested $[15,16]$. Only one other BCC strain was found to be lysogenized with KS10, B. cenocepacia strain C5424, and this strain has been shown previously to contain an integrated BcepMu [8]. In addition, 27 BCC clinical isolates obtained from the University of Alberta Hospitals (Pediatric/Adult) Cystic Fibrosis Clinic were also tested, revealing that KS10 is a prophage in seven of these isolates. All seven isolates were characterized as B. cenocepacia based on fur gene sequence analysis, suggesting that KS10 is a temperate phage specific to $B$. cenocepacia of the BCC (Table 1) [17].

\section{Integration of KSIO}

We attempted to identify the exact location of KS10 in all known BCC hosts by locating the actual phage integration site. Arbitrary primers (ARB6 and ARB2) and a specific KS10 primer, in addition to the APA gene Gold genome walker kit (Bio S\&T, Montreal), were used in PCR experiments to determine the precise insertion site of the integrated prophage. Unfortunately, PCR products obtained were found by sequence analysis to be the result of mispriming. This finding limited our ability to identify an exact site of insertion for KS10 in each B. cenocepacia genome, even though PCR tests unambiguously identified its presence in the genomes of these strains (Table 2).

Normally, KS10 will form plaques on lawns of B. ambifaria LMG 19467 and B. cenocepacia PC184, but at a lower frequency KS10 will also enter into its lysogenic life cycle. To demonstrate that KS10 can insert into the chromosomes of these hosts, colonies of strains that had been lysogenized with KS10 were selected. In order to obtain these lysogens, KS10 was plated with LMG 19467 and PC184 and turbid plaques indicative of lysogeny were selected using a needle tip. Two LMG 19467 lysogens and one PC184 lysogen were collected and demonstrated by PCR to harbour KS10.

\section{KSIO potential genes and homologues}

GeneMark and NCBI's ORF Finder programs were used to identify open reading frames within the KS10 genome sequence [18]. Each identified ORF was characterized

Table I: BCC strains/isolates testing positive for KS I0 prophage.

\begin{tabular}{|c|c|c|}
\hline Host (B. cenocepacia unless noted otherwise) & Source & Integration Site \\
\hline $\mathrm{K} 56-2$ & BCC experimental strain panel & $\begin{array}{l}\text { Transcriptional regulator; GntR family with } \\
\text { aminotransferase }\end{array}$ \\
\hline $\mathrm{J} 2315^{*}$ & BCC experimental strain panel & Oxidoreductase on Chromosome I \\
\hline C5424* & BCC experimental strain panel & ND \\
\hline $\mathrm{R} / 882$ & Clinical Isolate & ND \\
\hline $\mathrm{R} / 883$ & Clinical Isolate & ND \\
\hline $\mathrm{R} 1884$ & Clinical Isolate & ND \\
\hline SII528 & Clinical Isolate & ND \\
\hline $\mathrm{R} 1434$ & Clinical Isolate & ND \\
\hline $\mathrm{R} 750$ & Clinical Isolate & ND \\
\hline $\mathrm{R} 2314$ & Clinical Isolate & ND \\
\hline B. ambifaria LMG I9467 (lys I) & $\begin{array}{l}\text { BCC experimental strain panel } \\
\text { (modified, this study) }\end{array}$ & ND \\
\hline B. ambifaria LMG 19467 (lys2) & $\begin{array}{l}\text { BCC experimental strain panel } \\
\text { (modified, this study) }\end{array}$ & ND \\
\hline PCI 84 (lys3) & $\begin{array}{l}\text { BCC experimental strain panel } \\
\text { (modified, this study) }\end{array}$ & ND \\
\hline
\end{tabular}

$*$ - Also known to contain BcepMu

ND - Not determined 
(a) KS10 prophage in $\mathbf{J} 2315$

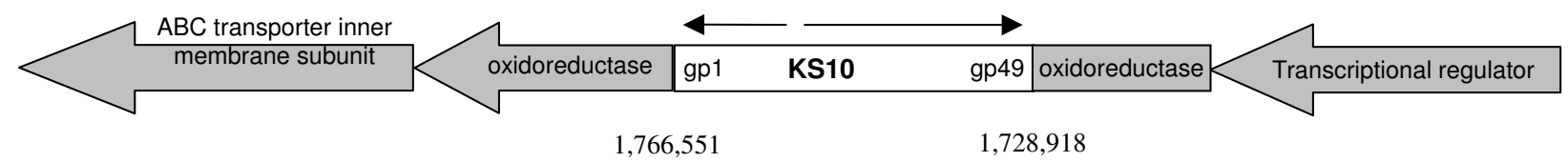

(b)

Left end Host/Phage DNA junction in J2315

aagagctgcgegatccegccGAGAGGGGGGACGTTTAGTT

Right end Phage/Host DNA junction in J2315

AAATTAAACGGCGCGCTACTcgecgttcgcatgcacgctc

(c) Putative transposase binding sites

\begin{tabular}{rll}
\hline \multicolumn{1}{l}{ Position } & Name & KS10 sequence \\
\hline 9 & L1 & GACGTTTAGTTTGAG \\
113 & L2 & GACGTTTAATTTGAC \\
& & \\
37592 & R2 & GTCAAATTAAACGTC \\
37614 & R1 & GTCAAATTAAACGGC \\
\hline $\begin{array}{r}\text { Consensus } \\
\text { sequence }\end{array}$ & gTCAAAtTAAACGtC \\
\hline
\end{tabular}

Figure 2

Phage/Host DNA junction and putative transposase binding sites. (A) KSIO has integrated into an oxidoreductase gene of $B$. cenocepacia J23 I5. Within the J23I5 genome, bases I,728,8I9 through I,766,55I are KSI0 prophage sequence. Direction of transcription of surrounding genes is indicated with thick arrows, while the direction of transcription of KSIO genes is indicated with thin arrows. The diagram is not drawn to scale. (B) Uppercase letters indicate KSIO sequence while lowercase represent $B$. cenocepacia J23 I5 host DNA. Phage right end is on the left in this diagram due to its orientation in the J23 I 5 genome, while the phage left end is on the right. (C) Four putative transposase binding sites at the terminal ends of KSI0. The position and sequence of the four 15 nucleotide direct repeats that are predicted to be TnpA binding sites in KSIO are indicated. The $\mathrm{LI}$ and $\mathrm{L} 2$ transposase binding sites, at the left end, are inverted relative to the RI and RI binding sites at the right end. All sequences are written 5'-3'. Consensus sequence is written in the direction of the RI and R2 binding sites.

using BLAST analysis against deposited sequences in the NCBI databases in order to assign a possible function (Table 2). Regions upstream of the ORFs were examined to determine potential ribosome binding sites (RBS) between 4 and 14 bases upstream of the start codon [19]. A total of forty-nine putative genes were identified, with just less than half encoding hypothetical proteins possessing no known function. KS10 genes 11 and 12 have significantly lower GC content than the genome overall (Table 2) suggesting that these genes were acquired by KS10 more recently. All putative genes utilize AUG as their translational start codon, except for two possessing a UUG and GUG start codon.
BLAST analysis (NCBI) showed that approximately $18 \%$ of KS10 proteins are homologous to proteins of BcepMu, whereas another $18 \%$ show homology to $\mathrm{Mu}$ proteins [20]. 14\% of KS10 proteins show identity to proteins from Ralstonia solanacearum strain UW551. Although they are not annotated as such in the UW551 GenBank entry, these are likely proteins of a prophage and not bacterial proteins. The highest percent identity that KS10 protein sequences exhibit to an orthologous protein is $67 \%$, while the majority of KS10 proteins show only moderate identity and similarity to other phage proteins (Table 2). Because DNA recombination can often occur between prophages in the same bacterial genome, it is perhaps sur- 
Table 2: Phage KSIO putative genes and homologues

\begin{tabular}{|c|c|c|c|c|c|c|c|c|c|c|}
\hline GP & Coding & Str. & $\begin{array}{c}\text { Possible RBS* \& Start } \\
\text { Codon }\end{array}$ & AA & Putative Function & Alignment Region & \% Identity & Score & $\% \mathbf{G C}$ & $\begin{array}{l}\text { Sig. matches to } \\
\text { proteins in NCBI's } \\
\text { GenBank }\end{array}$ \\
\hline 1 & $205-1437$ & - & AGGCGcgtaaATG & 410 & virion morphogenesis & $\begin{array}{c}400(28-418) / 248(16- \\
263)\end{array}$ & $30 \% / 33 \%$ & $160 / 137$ & 63.26 & $\begin{array}{l}\text { Pseudomonas } \\
\text { bacteriophage B3 gp33/ } \\
\text { BcepMu gp30 }\end{array}$ \\
\hline 2 & $1438-1698$ & - & AAAGGAGccaacaATG & 86 & hyp. protein & - & - & - & 61.69 & no sig. match \\
\hline 3 & $1743-3278$ & - & GGAAG//36 bp//cgtttATG & 511 & portal & $354(35-374)$ & $32 \%$ & 182 & 62.57 & BсерMu gp29 \\
\hline 4 & $3268-4884$ & - & AAGAGGcctgatccacgATG & 538 & $\begin{array}{l}\text { terminase large } \\
\text { subunit** }\end{array}$ & $520(4-521)$ & $55 \%$ & 569 & 58.94 & D3112 p26 \\
\hline 5 & $489 I-5388$ & - & AAGGGttgacgcATG & 165 & $\begin{array}{l}\text { terminase small } \\
\text { subunit** }\end{array}$ & $164(I-164)$ & $37 \%$ & 161 & 59.24 & D31 I 2 p24 \\
\hline 6 & $5392-5682$ & - & AGGGTATccgcgATG & 96 & hyp. protein & - & - & - & 59.45 & no sig. match \\
\hline 7 & $5679-5903$ & - & GGATAacgATG & 74 & $\begin{array}{l}\mathrm{dksA} / \text { traR C4-type zinc } \\
\text { finger }\end{array}$ & $74(I-72)$ & $45 \%$ & 44.3 & 60.89 & $\begin{array}{l}\text { Enterobateria phage } \\
\text { WPhi gp82 }\end{array}$ \\
\hline 8 & $5893-6186$ & - & GAGGAtgcccgtcATG & 102 & hyp. protein & - & - & - & 65.99 & no sig. match \\
\hline 9 & $6466-7137$ & - & GTGGGcctcgcagcATG & 223 & endolysin & $186(20-203)$ & $54 \%$ & 188 & 65.03 & BcepMu gp22 \\
\hline 10 & $7134-7535$ & - & GGGTGccgccgtcgcgaATG & 133 & holin & $\begin{array}{c}119(1-111) / 116(8- \\
113)\end{array}$ & $36 \% / 39 \%$ & $72.4 / 63.5$ & 58.96 & $\begin{array}{l}\text { Ralstonia solanacearum } \\
\text { UW55I***/BcepMu gP2 I }\end{array}$ \\
\hline 11 & $7650-8258$ & - & GGGAAGcgcgaaATG & 202 & hyp. protein & $\mid 77(53-2 \mid 1)$ & $26 \%$ & 45.4 & 53.53 & $\begin{array}{l}\text { Pseudomonas entomophila } \\
\text { L48 }\end{array}$ \\
\hline 12 & $825 I-8784$ & - & GGGAATAtgtaaagcATG & 177 & hyp. protein & - & - & - & 54.87 & no sig. match \\
\hline 13 & 8839-9309 & - & AAGAGGccgaccATG & 156 & $\begin{array}{l}\text { hyp. protein/phage } \\
\text { repressor }\end{array}$ & $\begin{array}{c}131(1-125) / 137(2- \\
127)\end{array}$ & $39 \% / 36 \%$ & $85.1 / 72.4$ & 56.69 & E. coli BI7I/BcepMu gp I7 \\
\hline 14 & $9391-9582$ & + & GGAGcaaatATG & 63 & $\begin{array}{l}\text { DNA binding protein/ } \\
\text { conserved hyp. protein }\end{array}$ & $\begin{array}{c}62 \\
(5-65) / 59(I-57)\end{array}$ & $33 \% / 52 \%$ & $38.1 / 65.1$ & 58.85 & $\begin{array}{l}\text { BcepMu gp |6/Ralstonia } \\
\text { solanacearum UW55 I }\end{array}$ \\
\hline 15 & $9579-10631$ & + & GGGGAGGtggATG & 350 & conserved hyp. protein & $\begin{array}{c}|4|(49-186) / / 27(77- \\
203)\end{array}$ & $28 \% / 28 \%$ & $38.1 / 44.7$ & 64.71 & $\begin{array}{l}\text { BcepMu gp I0/Ralstonia } \\
\text { solanacearum UW55 I }\end{array}$ \\
\hline 16 & $1067 \mid-12296$ & + & AGGTGcgatATG & 541 & transposase & $539(24-559)$ & $36 \%$ & 336 & 65.19 & $\begin{array}{l}\text { Ralstonia solanacearum } \\
\text { UW55I }\end{array}$ \\
\hline 17 & $12306-13298$ & + & GAATAAGGAGtgaccATG & 330 & transposition protein & $309(7-311)$ & $42 \%$ & 222 & 64.15 & $\begin{array}{l}\text { Ralstonia solanacearum } \\
\text { UW55I }\end{array}$ \\
\hline 18 & 13306-13494 & + & GTGAGGccatcATG & 62 & hyp. protein & - & - & - & 66.14 & no sig. match \\
\hline 19 & $1349 \mid-13796$ & + & GAGGTGAcggcATG & 101 & hyp. protein & - & - & - & 69.28 & no sig. match \\
\hline 20 & $|3859-| 437 \mid$ & + & TGGccgacgacagcATG & 170 & hyp. protein & $172(55-187)$ & $60 \%$ & 180 & 67.25 & $\begin{array}{l}\text { Ralstonia solanacearum } \\
\text { UW55I }\end{array}$ \\
\hline 21 & |4368-|4994 & + & AAGGAAcccatcATG & 208 & conserved protein & $\begin{array}{c}200(9-210) / 199(6- \\
204)\end{array}$ & $72 \% / 55 \%$ & $210 / 205$ & 63 & $\begin{array}{l}\text { Ralstonia solanacearum } \\
\text { UW55I/BcepMu gp05 }\end{array}$ \\
\hline 22 & $15005-15397$ & + & GAGGGGctggccATG & 130 & hyp. protein & - & - & - & 66.16 & no sig. match \\
\hline 23 & $15460-15732$ & + & AGGAGAAAcaccctcATG & 90 & $\begin{array}{l}\text { DNA binding protein } \\
\text { Hu-beta }\end{array}$ & $90(I-90)$ & $57 \%$ & 108 & 64.47 & $\begin{array}{l}\text { Bordetella pertussis } \\
\text { Tohama I }\end{array}$ \\
\hline 24 & $15809-16642$ & + & GAGGAAccccaaaATG & 277 & hyp. protein & $\begin{array}{c}\mid I 7(5|| 67) / 58(344- \\
40 I)\end{array}$ & $53 \% / 67 \%$ & 114 & 62.83 & $\begin{array}{l}\text { Burkholderia thailandensis } \\
\text { E264 gp38 }\end{array}$ \\
\hline 25 & $16806-17126$ & + & GGGGAGTGAcactgtgATG & 106 & hyp. protein & $111(9-119)$ & $37 \%$ & 55.1 & 64.49 & $\begin{array}{l}\text { Burkholderia pseudomallei } \\
\text { K } 96243\end{array}$ \\
\hline 26 & $17128-17568$ & + & GGAGGcccgctgacATG & 146 & $\begin{array}{l}\text { modulation of host } \\
\text { genes? }\end{array}$ & $129(9-135)$ & $30 \%$ & 58.5 & 64.17 & $\begin{array}{l}\text { Escherichia coli B7A/Mu } \\
\text { gp } 16\end{array}$ \\
\hline 27 & $17565-17963$ & + & GAAACGAccgcATG & 132 & $\begin{array}{l}\text { middle operon regulator } \\
\text { (Mor)**** }\end{array}$ & $106(14-118)$ & $31 \%$ & 52.8 & 64.91 & $\begin{array}{l}\text { Pseudomonas entomophila } \\
\text { L48 }\end{array}$ \\
\hline
\end{tabular}


Table 2: Phage KSI 0 putative genes and homologues (Continued)

\begin{tabular}{|c|c|c|c|c|c|c|c|c|c|c|}
\hline 28 & $18120-19322$ & + & AGAGAAccatTTG & 400 & protease & $330(10-322)$ & $33 \%$ & 147 & 65.59 & BcepMu gp32 \\
\hline 29 & 18876-19322 & + & AGGAagccATG & 148 & scaffold & - & - & - & 67.11 & BcepMu gp33 \\
\hline 30 & 19368-19724 & + & AGAGGATtcacATG & 118 & conserved hyp. protein & $\begin{array}{c}77(41-117) / 80(32- \\
109)\end{array}$ & $54 \% / 42 \%$ & $72.4 / 44.3$ & 71.71 & $\begin{array}{l}\text { Bacteriophage B3 gp37/ } \\
\text { BcepMu gp35 }\end{array}$ \\
\hline 31 & 19775-20722 & + & GGAGctatccATG & 315 & major head subunit & $311(4-307)$ & $47 \%$ & 243 & 64.87 & Bacteriophage B3 gp34 \\
\hline 32 & $20797-2 \mid 186$ & + & AAGAGAGatcATG & 129 & hyp. protein & - & - & - & 66.92 & no sig. match \\
\hline 33 & $21183-21686$ & + & GAAGGGccgcaaATG & 167 & hyp. protein & - & - & - & 65.48 & no sig. match \\
\hline 34 & $21683-22114$ & + & GGATAGGTAcgggaaATG & 143 & virion morph. & $\begin{array}{c}|6|(8-\mid 56)|2|(7- \\
\mid 24)\end{array}$ & $31 \% / 36 \%$ & $161 / 52.4$ & 60.19 & $\begin{array}{l}\text { Thermus aquaticus } \\
\text { Y5IMC23/Escherichia coli } \\
53638 \text { (MuG) }\end{array}$ \\
\hline 35 & $22114-22716$ & + & GGTGAGGATGAtgtgATG & 200 & $\begin{array}{l}\text { phage-related } \\
\text { conserved hyp. protein }\end{array}$ & $165(1-164)$ & $24 \%$ & 41.2 & 61.36 & $\begin{array}{l}\text { Burkholderia thailandensis } \\
\text { MSMB43 }\end{array}$ \\
\hline 36 & $22700-22978$ & + & ACGGG//25 bp//gatATG & 92 & hyp. protein & - & - & - & 64.52 & no sig. match \\
\hline 37 & $23022-24500$ & + & AAGGGAcattcgacATG & 492 & tail sheath protein & $472(12-48 I)$ & $34 \%$ & 188 & 63.15 & $\begin{array}{l}\text { Prophage MuSo2 } \\
\text { (Marinomonas sp.)/MuL } \\
\text { (gp39) }\end{array}$ \\
\hline 38 & $24546-24917$ & + & AAGGGAGTGaaacATG & 123 & hyp. protein & - & - & - & 62.9 & no sig. match \\
\hline 39 & $25000-25554$ & + & TGAGATTcccaccATG & 184 & tail assembly chaperone & - & - & - & 62.88 & FluMu gp $4 I^{* *}$ \\
\hline 40 & $25603-28038$ & + & GAGGAAGAgacgATG & 811 & $\begin{array}{l}\text { tail tape measure } \\
\text { (TPI09 fam.) }\end{array}$ & $484(3-458)$ & $38 \%$ & 265 & 64.2 & $\begin{array}{l}\text { Burkholderia vietnamiensis } \\
\text { G4 }\end{array}$ \\
\hline 41 & $28038-29408$ & + & GGAGGAAcgaactgATG & 456 & DNA circulation protein & $455(3-4 \mid 2)$ & $27 \%$ & 150 & 65.43 & $\begin{array}{l}\text { Polaromonas sp. JS666/ } \\
\text { MuN (gp47) }\end{array}$ \\
\hline 42 & $294|4-3057|$ & + & TGAcccctATG & 385 & tail protein & $352(96-337)$ & $33 \%$ & 153 & 63.04 & $\begin{array}{l}\text { Polaromonas sp. JS666/ } \\
\text { MuP (gp44) }\end{array}$ \\
\hline 43 & $3057 I-31092$ & + & GGAGcaaactgATG & 173 & baseplate assembly & $60(36-188)$ & $30 \%$ & 63.5 & 61.88 & $\begin{array}{l}\text { Escherichia. coli 0157:H7 } \\
\text { str. EC450I/MuQ (gp45) }\end{array}$ \\
\hline 44 & $31177-31758$ & + & AGGccatcATG & 193 & tail protein & $99(25-112)$ & $47 \%$ & 70.9 & 64.6 & $\operatorname{MuV}(\mathrm{gP} 46)$ \\
\hline 45 & $31755-32876$ & + & GGAAAAcatcATG & 476 & tail protein & $306(24-325)$ & $36 \%$ & 144 & 65.86 & $\begin{array}{l}\text { Pseudomonas entomophila } \\
\text { L48/MuW (gp } 47 \text { ) }\end{array}$ \\
\hline 46 & $32879-33478$ & + & AGGAGTgaccGTG & 173 & tail protein & $197(5-192)$ & $26 \%$ & 57 & 65.17 & $\begin{array}{l}\text { Desulfovibrio vulgaris } \\
\text { subsp. vulgaris str. } \\
\text { Hildenborough }\end{array}$ \\
\hline 47 & $33478-34455$ & + & GGAcatcgactgATG & 325 & tail fiber protein & $229(310-534)$ & $42 \%$ & 140 & 62.47 & $\begin{array}{l}\text { Burkholderia multivorans } \\
\text { ATCC } 17616\end{array}$ \\
\hline 48 & $34463-36691$ & + & TGAGGcacgcATG & 742 & $\begin{array}{l}\text { ABC-type phosphate } \\
\text { transport sys. }\end{array}$ & $459(40-493)$ & $46 \%$ & 364 & 60 & $\begin{array}{l}\text { Pseudomonas stutzeri } \\
\text { Al50I }\end{array}$ \\
\hline 49 & $367 I I-37475$ & + & GAGGTAcaaATG & 254 & hyp. protein & $270(I-248)$ & $28 \%$ & 81.3 & 59.48 & $\begin{array}{l}\text { Klebsiella pneumoniae } \\
342 \text { KPK_4II4 }\end{array}$ \\
\hline
\end{tabular}

Abbreviations: GP, gene product; Str., strand; RBS, ribosome-binding site; AA, amino acid; hyp, hypothetical; sig, significant; subsp, subspecies;

*Putative RBS binding sites for gPI, gP4, and gP37 were identified using RBS finder.

**Function predicted using PSI-BLAST

***All R. solanacearum UW55I references refer to unfinished DNA segment NZ_AAKL0I000024.I

*****Function predicted using PHYRE database 
prising that none of the KS10 genes show higher homology to BcepMu genes, since KS10 was found to be a prophage in several $B$. cenocepacia strains that are also lysogenized with BcepMu.

The genes identified as encoding hypothetical proteins using the standard BLASTX searches were subjected to analysis with PSI-BLAST. This program uses the predicted amino acid sequences of each gene to detect putative conserved domains within the sequence [21]. Using this program, KS10 gp4 and gp5 were identified as being the large and small terminase subunits, which are predicted to be ATP-binding proteins involved in DNA packaging into the procapsid [22]. These putative terminase genes are physically located after the virion morphogenesis and portal genes, an order that is conserved in many phage genomes $[8,22]$. Similarly, gp39 was found to show homology to FluMu41 using PSI-BLAST, a protein that is thought to be analogous to Lambda $\mathrm{G}$, a tail assembly chaperone [23].

Higher order bioinformatic analysis was performed using in-house programs for protein domain identification and functional prediction [24]. These analyses confirmed the results obtained using BLAST analyses but provided little additional functional protein information (see Additional file 1). Potential virulence factor genes were not identified within the genome of KS10. However, there remain several genes encoding hypothetical proteins within the KS10 genome that cannot be excluded as potential virulence factors until their functions are determined.

\section{Head assembly genes}

In most phages, head assembly involves five major stages, each involving a number of phage encoded proteins. The first stage, initiation, involves the initiation of polymerization of phage coat proteins by minor head proteins. Next, during shell formation, the immature procapsid, comprised of coat proteins, is assembled around the scaffold protein, which is subsequently cleaved to form a mature procapsid. Following the maturation of the procapsid are the two final stages, DNA packaging and head completion [25]. In KS10, gp1-gp5 and gp28-gp34 are expected to be involved in head assembly based on homology. KS10 gp28 and gp29 are identified as the protease and scaffolding proteins, which are structurally important to this process. The scaffolding protein has been shown to be essential in assembling coat proteins, encoded by gene 31 in KS10, into the capsid shell [26]. Once this shell is formed, it is thought that the protease, encoded by KS10 gene 28, is involved in removing this scaffold. As is the case in many other phages, the scaffold gene of KS10 is embedded in frame with the protease gene.
The proteins involved in the actual packaging of the DNA into the procapsid are the terminases, portal, and major capsid, which in KS10 are identified as gp4, gp5, gp3, and gp31 respectively. KS10 gene 34 encodes a protein that shows homology to MuG, which is thought to be a tail protein, though its function is unclear [27]. This gene also shows no similarity to other known genes in other nonMu-like phages [22]. KS10 gene 1 encodes a protein putatively involved in virion morphogenesis since it exhibits identity to MuF, though its function remains unknown. There are also a number of genes encoding hypothetical proteins interspersed within these genes, which could encode minor proteins involved in forming the mature capsid.

\section{Host Cell Lysis}

Phage host cell lysis usually involves a number of proteins, including a holin and a lysin. In KS10, these proteins are encoded by gene 10 and gene 9, respectively. There are a variety of lysins that can be phage encoded, all of which are peptidoglycan hydrolases. Holin proteins are hydrophobic and associate with the cytoplasmic membrane of the bacteria, creating holes that allow the lysin to move into the periplasm. KS10 holin and lysin proteins show homology to the holin and lysin of BcepMu, but the lysins differ in the N-terminal region [8]. PSI-BLAST analysis revealed the lysin of KS10 to have a soluble lytic transglycosylase (Slt) domain of 117 amino acids, similar to that of BcepMu and other non-Mu phages such as T7. Most enzymes of this nature found in $E$. coli have been shown to catalyze the cleavage between $\mathrm{N}$-acetylmuramic acid and $\mathrm{N}$-acetylglucosamine, resulting in 1,6-anhydromuramic acid. In E. coli, these enzymes degrade the cell wall murein during bacterial morphogenesis [28]. In BcepMu this Slt domain is conserved over residues 46155, while in KS10 the domain is conserved over residues 70-170. Most phage lysins lack their own signal peptide (SP) sequence, and are under control of the holin for release. In a search for encoded SP sequences within the lysin gene of KS10, however, a potential SP sequence was identified using SignalP server http://www.cbs.dtu.dk/ services/SignalP-2.0. The cleavage site in KS10 gp10 was predicted to be between amino acids 44 and 45 . The Nterminal region of KS10 putative lysin protein is also fairly hydrophobic, further suggesting that this protein contains an SP, although this activity has not been confirmed experimentally. Although not a common phenomenon, lysins of other phages, such as the Oenococcus oeni phage fOg44, have been found to contain an SP sequence. In this phage, overexpresssion of the lysin (Lys44) in E. coli was lethal, which is uncommon for phage lytic enzymes. When the SP was deleted, overexpression of Lys44 was no longer toxic [29]. Other phage genes that encode second- 
ary lysis proteins such as Rz/Rz1 were not identified in KS10.

\section{Transposition}

The proteins involved in transposition of KS10, specifically those encoded by gene 16 and gene 17, show the most homology to a transposase and transposition protein found in the genome of $R$. solanacearum strain UW551. The KS10 transposase shows little homology to BcepMu and other Mu-like transposases that are closely related to the $S$. auereus transposon Tn552 [8]. The integrated KS10 lacks the 5'-TG...CA-3' dinucleotides at its termini, which are characteristic of $\mathrm{Mu}$-like prophage elements related to BcepMu carrying an Rve integrase catalytic core domain [8]. However, the KS10 transposase does exhibit $30 \%$ identity and $44 \%$ similarity with the transposase from the transposable Pseudomonas phage D3112 [30]. Interestingly, where the KS10 prophage is integrated, no evidence of direct repeats has been identified in the flanking BCC DNA. The putative transposase binding sites for KS10 are imperfect direct repeats of 15 nucleotides. They do not appear to be similar to those found in BcepMu or E. coli Mu, which correlates well with the transposase of KS10 not showing significant homology to transposase of Mu or BcepMu. However, the putative KS10 transposase binding sites do contain the characteristic repeated A nucleotides within its target sequence (Figure 2). Unlike the prophages $\mathrm{Mu}$, FluMu, Pnm1, and Sp18, which have 6 putative transposase binding sites within their genomes, there are only 4 identified in KS10 [18]. These putative sites in KS10 were identified after a search for the consensus TnpA binding sites in both $\mathrm{Mu}$ and BcepMu were not identified in the KS10 sequence (Figure 2).

Gyrase binding sites have been identified in Mu and some other Mu-like phages, and are thought to promote the replicative transposition process that occurs during Mu lytic growth. In $\mathrm{Mu}$ and FluMu this binding site is located between Mu gpG and Mu gpI [18]. A search for this site in KS10 revealed no similar sequence, though we predict that this binding site is present, as a previous study by Sokolsky and Baker revealed that gyrase is necessary in $\mathrm{Mu}$ replicative transposition [31]. By using a drug that inhibits gyrase, they concluded that gyrase activity is important for the lytic life cycle of phage Mu.

\section{Tail Assembly}

Unlike proteins involved in head assembly, lysis, and transposition, there is little known about the proteins involved in Mu tail assembly. The last approximately 14 $\mathrm{kb}$ of the KS10 genome is involved in tail assembly. Like other members of the Myoviridae family, Mu-like phages have contractile tails, made up of a contractile sheath outside of an inner tail tube. The phage baseplate is located at the end of the tail and is attached to the tail fibers, which are involved in attachment to the host cell. KS10 genes 37 and 41 encode the tail sheath and tail tape measure proteins, respectively. In both $\mathrm{Mu}$ and $\mathrm{BcepMu}$, the tail tube gene is found between the genes encoding the tail sheath and the tape measure. In KS10 there is a hypothetical protein of 123 amino acids that, using a BLAST analysis, shows no homology or conserved domains to any protein in the database. This gene is the correct length and in the expected location to encode a tail tube protein based on other similar phages, but there is no experimental evidence to support this claim.

Xu et al. suggest that there is a -1 (or -2 in $\mathrm{Mu}$ ) frameshift conserved amongst dsDNA tailed phages that occurs before the tail tape measure gene and after the major tail gene [32]. This frameshift occurs in many phages including $\mathrm{Mu}, \mathrm{FluMu}, \mathrm{P} 2$, lambda, and D3. We found no evidence of a frameshift region before the tape measure gene in KS10. Using PSI-BLAST analysis, a FluM-like gp41 conserved domain was detected, which has been annotated as a Lambda $G$ analogue. In many dsDNA tailed phages there is a "slippery" sequence within this gene that causes a frameshift creating two overlapping ORFs. This sequence is usually a region of repeated $\mathrm{A}, \mathrm{T}$, or G nucleotides. In KS10, a sequence capable of causing this frameshift was not identified using both a manual search as well as the Frame Shift Finder program http://chain mail.bio.pitt.edu/ junxu/webshift.html.

KS10 gp42 to gp45 are predicted to be involved in baseplate assembly since they show homology to Mu proteins with this function. How each protein is involved in baseplate assembly in $\mathrm{Mu}$ is yet unknown. KS10 gene 47 encodes a 325 amino acid protein showing $42 \%$ identity to a tail collar protein of a $B$. multivorans strain prophage and $27 \%$ identity to a tail fiber protein of a prophage in $B$. thailandensis strain E264. In many Mu-like phages, the gene or genes encoding tail fibers is/are relatively long. BcepMu, for example, has a tail fiber gene encoding a 786 amino acid protein, similar to the phage $\mathrm{P} 2$, which is much larger than the small gene in KS10 showing low identity to a tail fiber. KS10 gp48, a 742 amino acid protein at the end of the genome shows $46 \%$ similarity to the periplasmic component of an ABC-type phosphate transporter system of Pseudomonas stutzeri A1501. However, the region showing homology to the gene from $P$. stutzeri shows no conserved domains when analyzed using PSIBLAST and no homology to any other periplasmic component from an ABC- type phosphate transporter system, suggesting that this is probably not the function of this protein. Using the GTOP sequence homology search http://spock.genes.nig.ac.jp/ ngenome/adseqsch.html to compare KS10 gp48 to all viruses in the database, the amino acid sequence of KS10 gp48 showed homology to 
other phage proteins annotated as being involved in host specificity and putative tail-host specificity. Though this homology was relatively low (25\%), it suggests that this 742 amino acid protein is a tail fiber protein involved in recognizing the phage receptor on the host cell, and not part of an ABC-type phosphate transport system.

\section{Organization of the KSIO genome}

Unlike other sequenced phages similar to $\mathrm{Mu}$, the first 5 gene products of KS10 are involved in head assembly. Following these genes in the KS10 genome are a number of genes involved in host cell lysis, followed by genes involved in transposition/integration, followed by additional genes involved in head assembly, and finally genes involved in tail formation. Mu-like phages, as is the case with most related phages, are usually genetic mosaics of each other and are often arranged in modules so that genes encoding proteins that interact, such as capsid genes, will not be separated by nonhomologous events [33]. The KS10 genome, however, has genes responsible for capsid formation separated, with virion morphogenesis, portal, and terminase genes at the beginning of the genome (Figure 3). This is uncommon for a Mu-like phage, as Mu phages generally have genes for head assembly in the middle of the genome, in the late gene region, which is usually more highly conserved than the early and middle gene regions [22]. To the best of our knowledge, this organization (dividing the capsid module) is unique to KS10. The other genes involved in head assembly, such as the protease, scaffold, and a MuG homologue, are located in the middle of the genome as expected.
When the KS10 genome is compared with the genome of $\mathrm{Mu}$, the first approximately $17 \mathrm{~kb}$ of KS10 genome is the most varied, and appears to be inverted (Figure 4), with genes responsible for host cell lysis located between integration and head assembly genes. The organization and direction of transcription in KS10 allows the genes responsible for integration and transcription regulation to interrupt the head assembly module. It is unknown why this phage, found in multiple $B$. cenocepacia genomes, would have its genome arranged this way. Previous theories of phage evolution imply that evolution by illegitimate recombination usually occurs by recombination events that will not interrupt the individual modules, as is seen in the KS10 genome [33]. Since the protease and scaffold genes are found in the middle of the genome, it is unlikely that the head assembly proteins encoded earlier would be used until these genes have been transcribed and translated. The module encoding proteins for tail assembly is located at the right end of the genome, similar to $\mathrm{Mu}$, though it lacks the invertible $\mathrm{G}$ region of $\mathrm{Mu}$. This invertible region found in $\mathrm{Mu}$ and some related phages, encodes the proteins involved in tail fiber synthesis, and the orientation of the region determines the host range of the phage [34]. BcepMu, a similar phage found in B. cenocepacia, has a right end similar to P2 and also lacks this invertible $\mathrm{G}$ region [7]. Although KS10 tail protein gene sequences do show homology to $\mathrm{Mu}$ tail protein gene sequences, KS10 also lacks this invertible G region.

Often phages contain genes that they have acquired through nonhomologous recombination with a host or

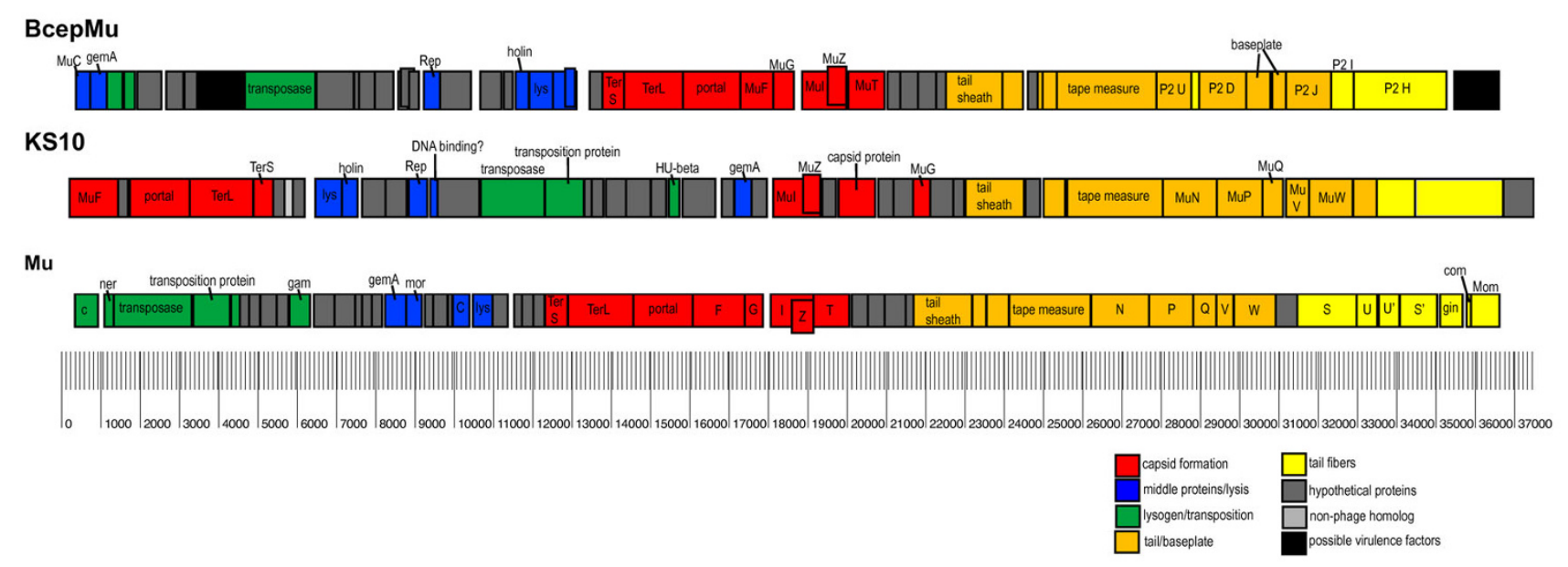

Figure 3

Genome maps of KSI 0 and related phages BcepMu and Mu (derived from NC_005882 and NC_000929, respectively). Each box represents a predicted gene drawn to scale using GenVision program (DNASTAR) and arrows indicate direction of transcription. Homologues and known phage proteins are indicated (Table I). Different colors represent different modules. Dark grey boxes indicate genes with no known phage homologues and are annotated as hypothetical proteins. 


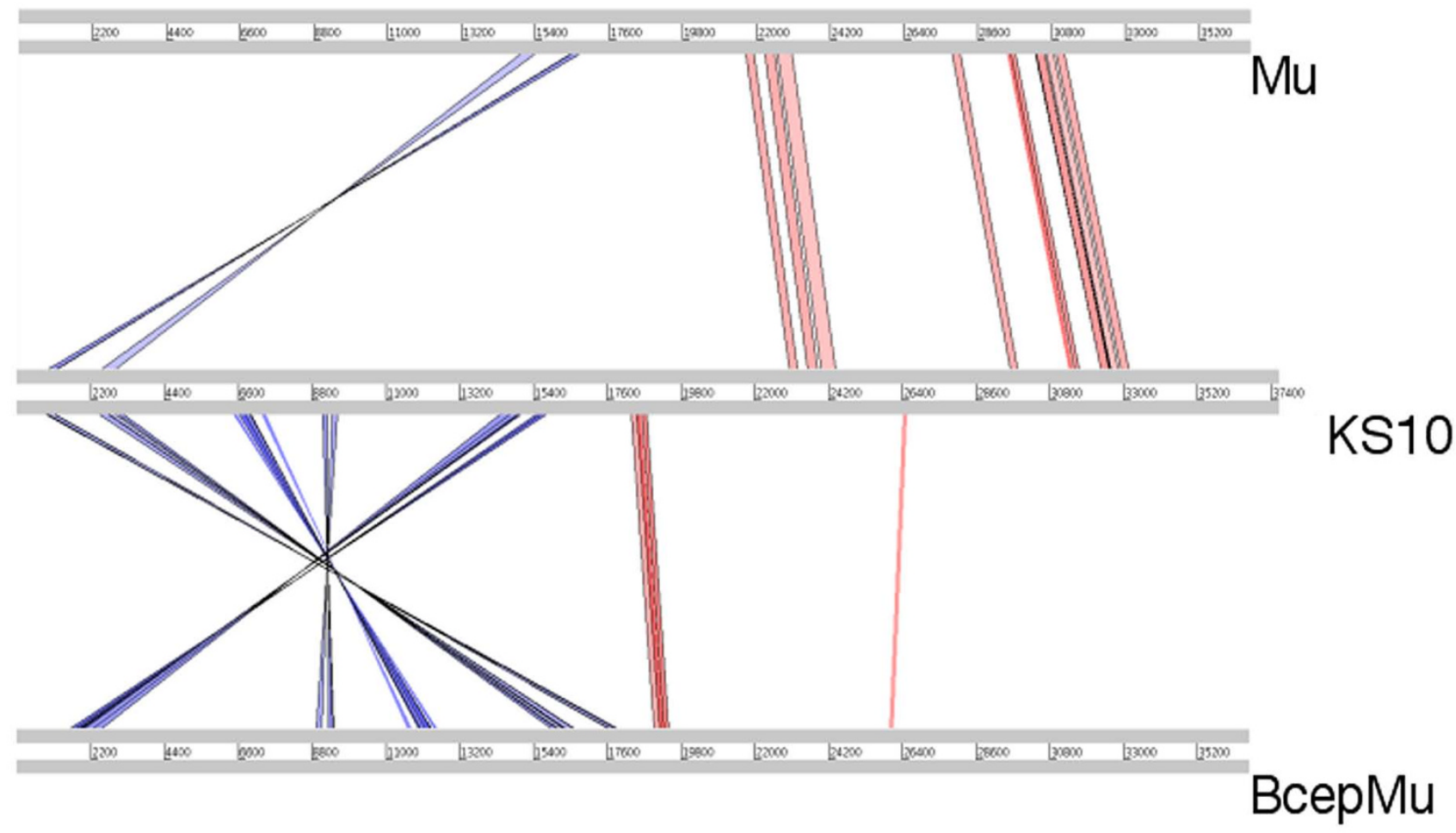

Figure 4

Mu relationships identified using Artemis Comparison Tool program. Translated BLAST (tblastx) was used to align translated genomic sequences of phages KSIO, BcepMu, and Mu. An E-value cutoff of 10 and a score cutoff of 40 were used in this comparison. Nucleotide basepairs are indicated between grey lines for each phage genome. The blue and red lines represent the reverse and forward matches, respectively, and color intensity is proportional to the sequence homology.

another phage. These acquired genes may encode proteins that can be involved in lysogenic conversion of the host cell, causing the bacterial host to become more virulent [33]. In order to test the hypothesis that KS10 contains genes whose products may increase the virulence of the bacterial host, we compared the killing effect of $B$. ambifaria LMG 19467 versus two strains of B. ambifaria 19467 lysogenized with KS10 in the recently developed BCC Galleria mellonella infection model [35]. We also tested wild type and KS10 lysogenized B. cenocepacia PC184 in this model. In all instances, the virulence of the bacterial strain harbouring the KS10 prophage was similar to that of the bacterial strains without the prophage, suggesting that KS10 does not carry virulence factor genes that are expressed in vivo (data not shown).

A gene in KS10 that shows no homology to other known phage proteins is gene 7 . KS10 gp7 shows relatively high identity to a dksA/traR C4-type zinc finger protein found in bacteriophage L-413C. DksA is a DnaK suppressor protein, which acts by suppressing transcription of DnaK, while TraR is a transcriptional activator. The coliphages
P2, 186, and phage Phi MhaA1-PHL101 also encode a protein showing homology to this dskA/traR protein, though other phages similar to KS10 do not. In Phi MhaA1-PHL101 the conserved Dsk/TraR region is extends over the last 40 amino acids and it is hypothesized to be involved in transcriptional activation [36]. In KS10 gp7 the domain is conserved across the whole protein. This gene is located upstream of the first module of head genes. A possible role for this protein in KS10 could be to repress the transcription of genes 1-5 until the second head module is transcribed. If so, this protein may be responsible for controlling and coordinating the transcription of the two head gene modules.

While the KS10 genome seems to show unusual variability in its genomic organization, especially in the first half, it is possible that there are many other phages with similar organizations that have not yet been sequenced. Phages are one of the most abundant particles on Earth, but there are only a relatively small number of these phages whose genomes have been sequenced, making it difficult to draw definitive conclusions about the relationship of KS10 and 
its gene products to other phages. However, complete sequencing of bacterial genomes has provided increasing opportunities for prophage identification, and has also produced incontrovertible evidence of extensive phagemediated exchange of genetic material between species. Although previous publications have suggested that $\mathrm{Mu}-$ like prophage elements are either rare or sufficiently divergent to be unrecognizable by sequence comparison, we have shown that polylysogeny does indeed occur in the BCC and that polylysogeny can occur with two different Mu-like BCC phages $[8,12]$. Further studies are required to understand the interactions of multiple active phages within a single genome of a strain of the BCC and their influence on cellular lifestyle and bacterial pathogenicity. The characterization of broad-host range BCC phages, regardless of whether they are lytic or lysogenic, will provide an opportunity to further develop these phages as novel therapeutic agents for use against infections caused by the highly antibiotic resistant BCC.

\section{Conclusion}

KS10 is a novel 37,635 bp phage of the opportunistic bacterial pathogen Burkholderia cenocepacia. Genome sequence analysis and annotation of this phage reveals that KS10 shows the closest sequence homology to the transposable phages $\mathrm{Mu}$ and BcepMu. KS10 differs from most phages in that its capsid genes are arranged into two modules, giving evidence of one or more prior genetic rearrangements. KS10 was found to be a prophage in three different strains of $B$. cenocepacia, including strains K56-2, $\mathrm{J} 2315$, and C5424, and seven tested clinical isolates of $B$. cenocepacia, but no other BCC species. A survey of 23 strains and 20 clinical isolates of the BCC revealed that KS10 is able to form plaques on lawns of $B$. ambifaria LMG 19467, B. cenocepacia PC184, and B. stabilis LMG 18870. There were no potential virulence factors identified in KS10, though many hypothetical proteins were identified with no known function.

\section{Methods}

\section{Bacterial strains, phages, and media}

BCC strains including B. cenocepacia from the BCC experimental strain panels were obtained from the Belgian Coordinated Bacteria Collection M (Ghent, Belgium), or the Canadian Burkholderia cepacia complex Research and Referral Repository (Vancouver, Canada) $[15,16]$. BCC clinical isolates were obtained from the University of Alberta Hospitals Cystic Fibrosis Clinic (Edmonton, Canada). 1/2 concentration Luria Bertani (LB) broth or solid media was used to grow BCC host strains. LB solid media supplemented with ampicillin $(0.1 \mathrm{~g} / \mathrm{L})$ was used to grow competent E. coli strains harbouring pUC19 or pGEM7Z. Growth of BCC strains was carried out aerobically $30^{\circ} \mathrm{C}$ overnight, while E. coli DH5 $\alpha$ used for cloning was grown aerobically overnight at $37^{\circ} \mathrm{C}$. KS10 plaques were picked from uninduced lawns of K56-2 and stored at $4{ }^{\circ} \mathrm{C}$ in suspension media (SM) (50 mM Tris/HCl, pH 7.5, $100 \mathrm{mM}$ $\mathrm{NaCl}, 10 \mathrm{mM} \mathrm{MgSO}_{4}$, and $0.01 \%$ gelatin solution). Phage stocks were prepared by placing an agar plug containing a single plaque into $1 \mathrm{~mL}$ of SM using a sterile glass Pasteur pipette and stored at $4^{\circ} \mathrm{C}$.

\section{Bacteriophage production and host range testing}

To determine the titer of one KS10 plaque in $1 \mathrm{ml}$ of SM, the lysate was serially diluted in SM and plaque forming units (pfu) were determined using the soft agar overlay method. To determine host ranges, the KS10 phage stock was mixed with 23 different individual strains of BCC and 27 clinical isolates of BCC in soft agar overlays and plaques were tallied after 18-22 hours growth. KS10 distribution within the species of the BCC was determined using a PCR assay with oligonucleotide primers F3 (5'CCGATTCCCACATCACGATCC) and R3 (5'-TGCGGGCATTTCAGCTTTCG). Bacterial genomic DNA was prepared as previously described [37]. PCR was performed in $50 \mu \mathrm{l}$ reactions containing approximately $20 \mathrm{ng}$ of template DNA and 25 pmol of each primer using TaqPCRx DNA Polymerase, Recombinant (Invitrogen) for one cycle at $96^{\circ} \mathrm{C}$ for one minute, 30 cycles at $96^{\circ} \mathrm{C}$ for 30 seconds, $70^{\circ} \mathrm{C}$ for 30 seconds, $72^{\circ} \mathrm{C}$ for 1 minute, and one cycle at $72^{\circ} \mathrm{C}$ for two minutes. To ensure the authenticity of the KS10 product, PCR products were analyzed by agarose gel electrophoresis and purified for sequencing using a QIAquick PCR purification kit (Qiagen Inc., Mississauga, Ont.).

\section{Transmission electron microscopy (TEM)}

KS10 was obtained from an overnight culture of K56-2 $\left(\mathrm{OD}_{600}\right.$ of approximately 2.0). Culture was centrifuged at $10,000 \mathrm{rcf}$ for 2 minutes and filter sterilized using $0.45 \mu \mathrm{m}$ filters. Filtrate was spotted onto copper grids and stained with $2 \%$ phosphotungstic acid. Micrographs were obtained using a Philips/FEI (Morgagni) Transmission Electron Microscope with CCD camera (Microscopy Unit, Biological Sciences, University of Alberta).

\section{Phage DNA Isolation and Sequencing}

KS10 was propagated on host B. ambifaria LMG 19467 for DNA extraction. KS10 DNA was isolated from bacteriophage lysate using the Wizard Lambda DNA purification system (Promega Corp., Madison, WI). The purified KS10 DNA was digested using the restriction enzymes SphI, EcoRI, and XhoI (Invitrogen Corp., Carlsbad, CA). To create cloned phage genomic DNA libraries, phage DNA fragments were purified using GeneClean II kit (QBiogene) and ligated into pUC19 or pGEM7Z. Ligated plasmids were transformed into chemically competent E. coli DH5 $\alpha$ and the plasmids were purified for sequenc- 
ing using a Qiaprep miniprep kit (Qiagen Inc., Mississauga, Ont.). Sequencing of the KS10 inserts was carried out using the ABI BigDye Terminator Cycle Sequencing Kit (Applied Biosystems) with an ABI 3100 Gene Analyzer (Molecular Biology Service Unit, Biological Sciences, University of Alberta). Sequencing data was edited using EditView 1.0.1 (Applied Biosystems) and assembled using AutoAssembler (Applied Biosystems). GeneMark and NCBI's ORF Finder programs were used to detect possible open reading frames (ORFs) $[18,20]$. Where multiple start codons or ORFs were indicated, the presence of a potential ribosomal binding site (RBS) was used to help identify the most likely ORF. Each identified ORF was tested with BLASTX analysis http:// www.ncbi.nlm.nih.gov/BLAST to assign putative functions [20]. When BLASTX revealed no significant matches, PSI-BLAST (NCBI) was also used [21]. Comparisons of KS10 sequences with the B. cenocepacia J2315 genome sequence were carried out using the BLAST server at the Sanger Centre sequencing project web site http://www.sanger.ac.uk/projects/B cenocepacia).

Comparative maps were constructed using GenVision software (DNASTAR, Inc., Madison, Wis.) and Artemis Comparison Tool (Sanger Centre, UK) [38]. The complete annotated DNA sequence of bacteriophage KS10 can be found in GenBank under accession number EU822883.

\section{Characterization of PCI 84 and LMG 19467 lysogens}

KS10 was propagated on BCC strains PC184 and LMG 19467 using the soft agar overlay method. Turbid plaques were identified, picked using a twenty-gauge needle, and placed in $1 \mathrm{~mL}$ of $1 / 2 \mathrm{LB}$ in an incubating shaker overnight at $30^{\circ} \mathrm{C}$. Cultures were streaked for individual colonies that were then tested for their inability to support plaque formation by KS10. PCR using KS10-specific primers further confirmed the presence of a KS10 prophage. In an attempt to determine the integration sites of KS10 in the three isolated lysogens (as well as strain C5424 and the seven B. cenocepacia clinical isolates), PCR using arbitrary primers (ARB6 and ARB2) and a specific KS10 primer were used, and, in addition, the APA gene Gold genome walker kit (Bio S\&T, Montreal) was used according to the manufacturer's directions.

\section{Authors' contributions}

ADG, KHL, and KDS carried out the phage genome isolation and sequencing, ADG and KHL participated in the sequence alignment and annotation, and ADG drafted the manuscript. PS carried out additional sequence alignments and generated alignment figures. SS and DSW performed advanced bioinformatic analysis of the predicted genes and proteins. JJD conceived of the study, participated in its design and coordination, and edited the final drafts of the manuscript. All authors read and approved the final manuscript.

\section{Additional material}

\section{Additional file 1}

Additional properties of KS10 proteins gp1-gp49. The data provided represent advanced bioinformatic analyses of the predicted proteins encoded on the bacteriophage KS10 genome.

Click here for file

[http://www.biomedcentral.com/content/supplementary/14712164-9-615-S1.doc]

\section{Acknowledgements}

Support for this work was provided primarily from a grant from the Canadian Cystic Fibrosis Foundation. This work was also supported in part from a grant from the Canadian Institutes of Health Research for collaborative research funding to J.J.D and D.S.W. of the "CIHR Team in Aerosol Phage Therapy". K.H.L. and K.D.S. are supported by CGS-D and PGS-D scholarships, respectively, from the Natural Sciences and Engineering Research Council of Canada.

\section{References}

I. Burkholder WH: Sour skin, a bacterial rot of onion bulbs. Phytopathology 1950, 40:II5-II7.

2. Ramette A, LiPuman J], Tiedje JM: Species abundant and diversity of Burkholderia cepacia complex in the environment. Appl Environ Microbiol 2005, 7 I: I 193-I201.

3. Isles A, Maclusky I, Corey M, Gold R, Prober C, Fleming P, Levison H: Pseudomonas cepacia infection in cystic fibrosis: an emerging problem. J Pediatr 1984, 104:206-210.

4. Bottone EJ, Douglas SD, Rausen AR, Keusch GT: Association of Pseudomonas cepacia with chronic granulomatous disease. J Clin Microbiol 1975, I:425-428.

5. Seed KD, Dennis JJ: Isolation and characterization of bacteriophages of the Burkholderia cepacia complex. FEMS Microbiol Lett 2005, 25 I:273-280.

6. D'Herelle F: The bacteriophage: its role in immunity. Williams and Wilkens Co./Waverly Press, Baltimore, USA; 1922.

7. Hagens S, Habel A, von Ahsen U, von Gabain A, Blasi U: Therapy of Experimental Pseudomonas Infections with a Nonreplicating Genetically Modified Phage. Antimicrob Agents Chemother 2004, 48:3817-3822.

8. Summer EJ, Gonzalez CF, Carlisle T, Mebane LM, Cass AM, Savva CG, LiPuma J, Young R: Burkholderia cenocepacia phage BcepMu and a family of Mu-like phages encoding potential pathogenesis factors. J Mol Biol 2004, 340:49-65.

9. Waldor MK, Mekalanos J]: Lysogenic conversion by a filamentous phage encoding cholera toxin. Science 1996, 272:1910-1913.

10. O'Brien AD, Newland JW, Miller SF, Holmes RK, Smith HW, Formal SB: Shiga-like toxin converting phage from Escherichia coli strains that cause hemorrhagic colitis or infantile diarrhea. Science 1984, 226:694-696.

I I. McCloskey RV: Scarlet fever and necrotizing fascitis caused by coagulase-positive hemolytic Staphylococcus aureus, phage type 85. Annals of Internal Medicine 1973, 78:85-87.

12. Langley R, Kenna DT, Vandamme P, Ure R, Govan JRW: Lysogeny and bacteriophage host range within the Burkholderia cepacia complex. J Med Microbiol 2003, 52:483-490.

13. Nakagawa I, Kurokawa K, Yamashita A, Nakata M, Tomiyasu Y, Okahashi N, Kawabata S, Yamazaki K, Shiba T, Yasunaga T, Hayashi H, Hattori $M$, Hamada $S$ : Genome sequence of an $M 3$ strain of streptococcus pyogenes reveals a large-scale genomic rearrangement in invasive strains and new insights into phage evolution. Genome Research 2003, I3:1042-1055.

14. Canchaya C, Fournous G, Brüssow H: The impact of prophages on bacterial chromosomes. Mol Microbiol 2004, 53:9-18.

15. Mahenthiralingham E, Coenye T, Chung JW, Speert DP, Govan JR, Taylor P, Vandamme P: Diagnostically and experimentally use- 
ful panel of strains from the Burkholderia cepacia complex. I Clin Microbiol 2000, 38: I042-1047.

16. Coenye T, Vandamme P, LiPuma JJ, Govan JR, Mahenthiralingham E: Updated version of the Burkholderia cepacia complex experimental strain panel. J Clin Microbiol 2003, 41:2797-2798.

17. Lynch KH, Dennis J]: Development of a species-specific fur gene-based method for identification of the Burkholderia cepacia complex. J Clin Microbiol 2008, 46:447-455.

18. Besemer J, Borodovsky M: GeneMark: web software for gene finding in prokaryotes, eukaryotes and viruses. Nucleic Acids Res 2005, 33:W45I-454.

19. Harley CB, Reynolds RP: Analysis of E. coli promoter sequences. Nucleic Acids Res 1987, 15:2343-236I.

20. Altschul SF, Gish W, Miller W, Myers EW, Lipman DJ: Basic local alignment search tool. J Mol Biol 1990, 5:403-4I0.

21. Altschul SF, Madden TL, Schäffer AA, Zhang J, Zhang Z, Miller W, Lipman DJ: Gapped BLAST and PSI-BLAST: a new generation of protein database search programs. Nucleic Acids Res 1997, 25:3389-3402.

22. Morgan G], Hatfull GF, Casjens S, Hendrix RW: Bacteriophage Mu genome sequence: Analysis and comparison with Mu-like prophages in Haemophilus, Neisseria and Deinococcus. J Mol Biol 2002, 3 I 7:337-359.

23. Levin ME, Hendrix RW, Casjens SR: A programmed translational frameshift is required for the synthesis of a bacteriophage $\lambda$ tail assembly protein. J Mol Biol 1992, 234: I24-139.

24. Van Domselaar GH, Stothard P, Shrivastava S, Cruz JA, Guo A, Dong X, Lu P, Szafron D, Greiner R, Wishart DS: BASys: a web server for automated bacterial genome annotation. Nucleic Acids Res 2005:W455-459.

25. Grimaud R: Bacteriophage Mu head assembly. Virology 1996, 217:200-210.

26. King J, Casjens S: Catalytic head assembling protein in virus morphogenesis. Nature 1974, 25 I: I I2-I I9.

27. Giphart-Gassler M, Wijffelman C, Reeve J: Structural polypeptides and products of late genes of bacteriophage Mu: Characterization and functional aspects. J Mol Biol I98I, I45:139-163.

28. Hoeltje JV, Mirelman D, Sharon N, Schwarz U: Novel type of murein transglycosylase in Escherichia coli. J Bacteriol 1975, 124:1067-1076.

29. Wang IN, Smith DL, Young R: The protein clocks of bacteriophage infections. Annu Rev Microbiol 2000, 54:799-825.

30. Wang PW, Chu L, Guttman DS: Complete sequence and evolutionary genomic analysis of the Pseudomonas aeruginosa transposable bacteriophage D3II2. I Bacteriol 2004, 186:400-410.

31. Sokolsky TD, Baker TA: DNA gyrase requirements distinguish the alternate pathways of Mu transposition. Mol Microbiol 2003, 47:397-409.

32. Xu J, Hendrix R, Duda R: Conserved translational frameshift in dsDNA bacteriophage tail assembly genes. Mol Cell 2004, 16:|I-2|.

33. Hendrix RW: Bacteriophage genomics. Curr Opin Microbiol 2003, 6:506-5II.

34. De Putte P, Cramer S, Giphart-Gassler M: Invertible DNA determines host specificity of bacteriophage Mu. Nature 1980, 286:218-222.

35. Seed KD, Dennis J]: Development of Galleria mellonella as an alternative infection model for the Burkholderia cepacia complex. Infect Immun 2008, 76: I267-1276.

36. Highlander SK, Weissenberger S, Alvarez LE, Weinstock GM, Berget PB: Complete nucleotide seqeuence of a P2 family lysogenic bacteriophage, Phi MhaAl-PHLIOI, from Mannheimia haemolytica serotype AI. Virology 2006, 350:79-89.

37. Ausubel FM, Brent R, Kingston RE, Moore DD, Seldman JG, Smith JA Struhl K: Current protocols in molecular biology. Greene Publishing Associates, New York, NY; 1991.

38. Carver TJ, Rutherford KM, Berriman M, Rajandream MA, Barrell BG, Parkhill J: ACT: the Artemis Comparison Tool. Bioinf 2005, 2I:3422-3423.
Publish with Bio Med Central and every scientist can read your work free of charge

"BioMed Central will be the most significant development for disseminating the results of biomedical research in our lifetime. "

Sir Paul Nurse, Cancer Research UK

Your research papers will be:

- available free of charge to the entire biomedical community

- peer reviewed and published immediately upon acceptance

- cited in PubMed and archived on PubMed Central

- yours - you keep the copyright

Submit your manuscript here:

http://www.biomedcentral.com/info/publishing_adv.asp
BioMedcentral 